\section{Management of compound odontoma with guided bone regeneration. A case report.}

\author{
José David Aguilar ${ }^{1}$, Daniela Carolina Cabrera ${ }^{1}$, \\ Xavier Orlando Inga ${ }^{1} \&$ Andrea Catalina Terreros ${ }^{2}$.
}

Abstract: An odontoma is a mixed benign odontogenic tumor, being a hamartomatous neoplastic lesion of odontogenic epithelial and mesenchymal cells that form enamel, dentine and cement, which occurs in the second to third decade of life, and is commonly a radiographic finding. The aim of this paper is to describe the management of a compound odontoma with guided bone regeneration. A 20 -year-old asymptomatic male patient with no relevant surgical medical history underwent surgery for the enucleation of a compound odontoma followed by guided bone regeneration of the defect, and histopathological study of the specimen. The evolution was favorable, without any sign of recurrence. It is important to carry out a previous surgical plan, taking into account the size of the defect resulting from its enucleation, assess the need for guided bone regeneration and use complementary diagnostic resources for a satisfactory recovery.

Keywords: Odontoma, compound odontoma, benign tumor, odontogenic tumor.

\section{INTRODUCTION}

An odontoma is a mixed benign odontogenic tumor (Pro Lizuain et al., 2016; Brenes-Barquero, 2014; Hernández et al., 2016) which is comprised of a hamartomatous neoplastic lesion of odontogenic epithelial and mesenchymal cells that form enamel, dentine and cement (Demicheri et al., 2015; Reddy et al., 2014).

They are often asymptomatic, and are generally a radiological finding. This type of odontogenic tumor is more frequent in permanent dentition but not unusual in temporary dentition; odontomas are classified into two groups: compounds and complexes (Wright \& Vered, 2017; Neville et al., 2015).

Compound odontomas have identifiable dental tissues in an organized style, shaping structures similar to smaller teeth called denticle (Pro Lizuain et al., 2016; Brenes-Barquero, 2014;). Complex odontomas also have all of the dental tissues, but in a disorganized manner. Compound odontomas are more common (Hernández et al., 2016; Ducoing et al., 2014). Compound odontomas account for approximately $45 \%$ of odontogenic tumors (Pro Lizuain et al., 2016; Ducoing et al., 2014), and are slightly more common in men than in women (Thistle et al., 2016; Moya de Calderon, 2016, Palacios et al., 2016).

The etiology is unknown, but they are associated with different factors such as trauma (Brenes-Barquero, 2014; Hernández et al., 2016), infection and chronic inflammatory processes during teeth development
INT J MED SURG SCI

Affiliations: ${ }^{1}$ Universidad Católica de Cuenca - Sede Azogues, Ecuador. ${ }^{2}$ Práctica privada, Ecuador.

Corresponding author: José David Aguilar. Dirección: Av 16 de abril, Azogues, Ecuador. Phone: +593992898283.

E-mail: jdaguilarm@ucacue.edu.ec

Receipt:

$03 / 22 / 2018$

Revised:

$04 / 23 / 2018$

Acceptance:

06/14/2018

Online:

$08 / 30 / 2018$
Conflict of interests: None.

Ethics approval: Universidad Católica de Cuenca.

Funding: None.

Authors' contributions: All authors carried out the entire case.

Acknowledgements: None.

doi: $10.32457 /$ ijmss.2018.006. 
Demicheri et al., 2015; Reddy et al., 2014; Andrade et al., 2017), which generate odontoblastic hyperactivity and the reactivation of epithelial cell rests of Malassez (Thistle et al., 2016; Moya de Calderon, 2016). It is also been reported to be associated with alterations in the genes controlling tooth development, and associated with syndromes such as Gorlin-Goltz syndrome and Gardner syndrome (Ducoing et al., 2014; Palacios et al., 2016; Rojas, 2013).

The majority of compound odontomas are located in the area of the incisors and canines of the upper and lower maxilla, but more frequently in the upper maxilla (Andrade et al., 2017; Ducoing et al., 2014). Complex odontomas are located in the posterior region of the upper and lower jaws, with a higher presence in the lower jaw (Brenes-Barquero, 2014; Rojas, 2013). Extraosseous cases have also been described (Hernández et al., 2016)

Clinically, they are characterized by their slow and painless growth, generally between the second and third decade of life; their presence can be suspected in cases such as a delayed tooth eruption or by the bulging of bony cortices of the jaws (Palacios et., 2016). In some cases, they can cause pain, expansion of the bone tables, dental displacements, lip anesthesia and swelling (Pro Lizuain et al., 2016; Wright \& Vered, 2017).

Compound odontomas usually manifest as radiopaque images that simulate multiple teeth in a single focus (Pro Lizuain et al., 2016; Hernández et al., 2016; Andrade et al., 2017). In the case of composite odontomas, it can be observed from 4 to 28 structures, in a single group or capsule, or distributed in multiple capsules (Demicheri et al., 2015; Ducoing, 2014; Thistle et al., 2016).

Three phases of calcification can be identified, that is, three phases in which radiographic differences can be observed: Initial: radiolucent, due to the absence of calcification of the dental tissues; Intermediate: partial calcification; and Mature: classically radiopaque with a predominance of calcification of the tissues, which are surrounded by a radiolucent halo (Thistle et al., 2016; Palacios et al., 2016).

Histologically, they are comprised of different dental tissues such as enamel, dentin, cement and sometimes pulp tissue; these tissues may have a normal or abnormal ratio. Both types of tumors have a connective tissue capsule around them, which is similar to the follicle surrounding a normal tooth (Brenes-Barquero, 2014). They possess cells of ectodermal, mesenchymal and epithelial origin, which form a calcified or hypocalcified enamel, primary dentin, immature cement and pulp tissue contained within a capsule or in a fibrous matrix of connective tissue (Demicheri et al., 2015; Moya de Calderon, 2016; Palacios et al., 2016).

Some differential diagnoses include: complex odontoma, supernumerary teeth, cementoma, and calcifying epithelial odontogenic tumor, among others (Ducoing et al., 2014).

The common treatment of odontomas is surgical, which has a favorable prognosis, although an excisional biopsy is taken to later perform histopathological analysis (Pro Lizuain et al., 2016; . Reddy et al., 2014; Ducoing et al., 2014; Rojas, 2013).

The aim of this case report is to describe the management of a compound odontoma with guided bone regeneration.

\section{CASE REPORT}

A 20 year-old male patient, who was asymptomatic and without a relevant surgical medical history, attended the specialty clinic of the University of Cuenca at the Azogues headquarters, for the extraction of the mandibular and maxillary third molars by orthodontic referral.

At the clinical examination, the patient had caries, moderate fluorosis, mild periodontal disease and presented bulging in the right lower premolar region without any change to the coloration of the mucosa and a firm consistency (Figures 1 and 2). The patient did not report any previous painful symptoms nor had he noticed this bulge, so he did not know how to express its time of evolution.

The panoramic radiograph (Figure 3) shows small radiopaque areas with a radiolucent halo in the region of the second right lower premolar in tooth 4.5 , in its radicular portion between the middle third and apical third.

A presumptive diagnosis of compound odontoma was made. Then, a computed cone tomography (CBCT) of 

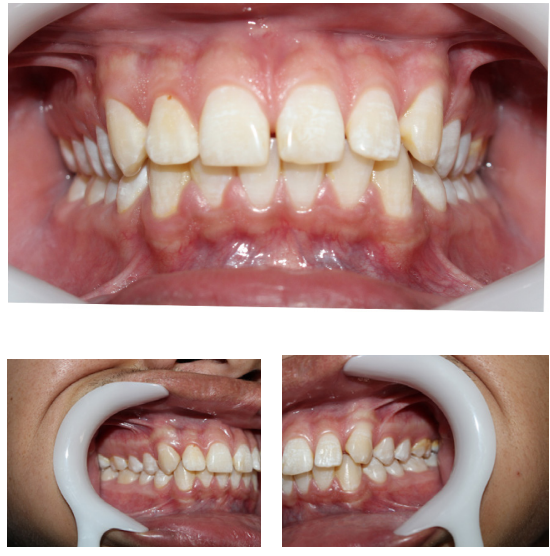

Figure 1: Frontal and lateral view of the oral region of the patient

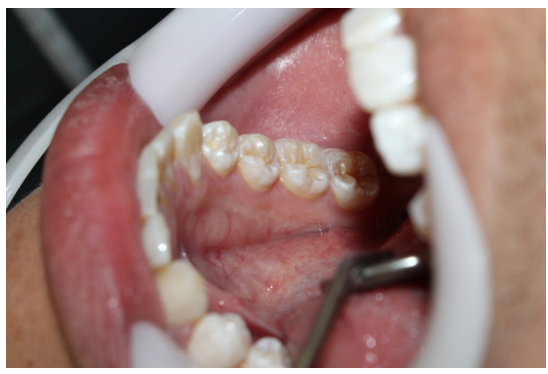

Figure 2: Bulging in the area of the lower right premolars and first molar

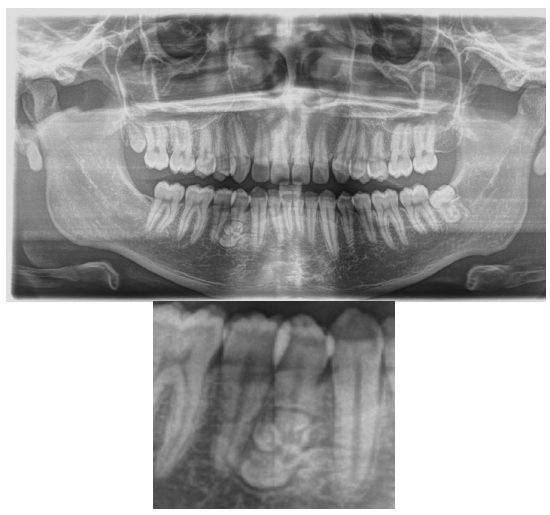

Figure 3: Panoramic radiography with emphasis on the lower right premolar area where several radiopacities with a defined radiolucent halo were found.

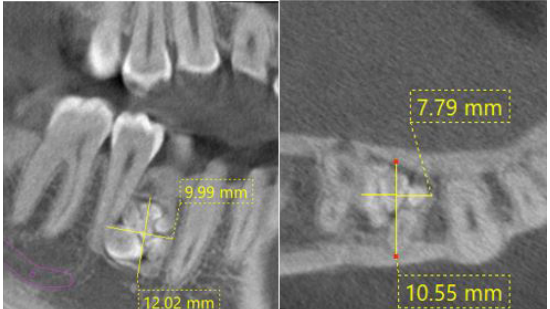

Figure 4: СВСТ of the area of interest, observing a sagittal section on the right figure and coronal section on the left figure, where several hyperdense zones with a hypodense halo were observed with the length of the Iesion.

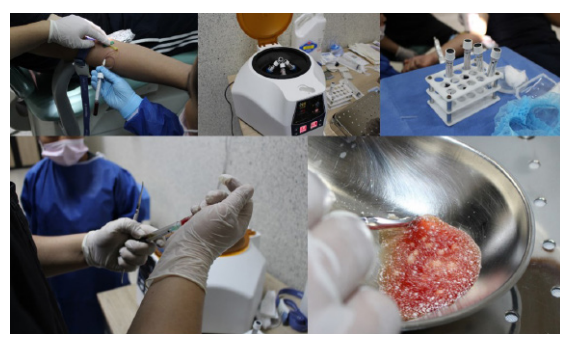

Figure 5:Platelet Rich Fibrin extraction for injection and mixture with a particulate bone allograft mixture.

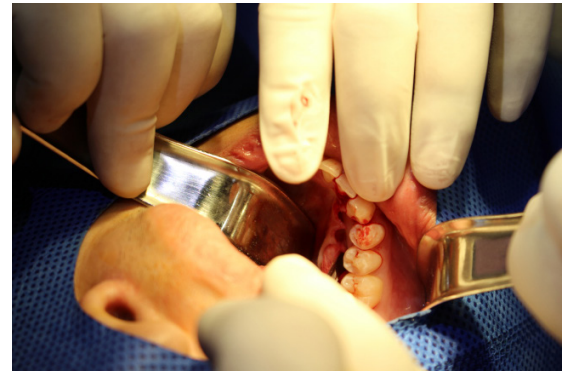

Figure 6: Incision and detachment of the mucoperiosteal flap 


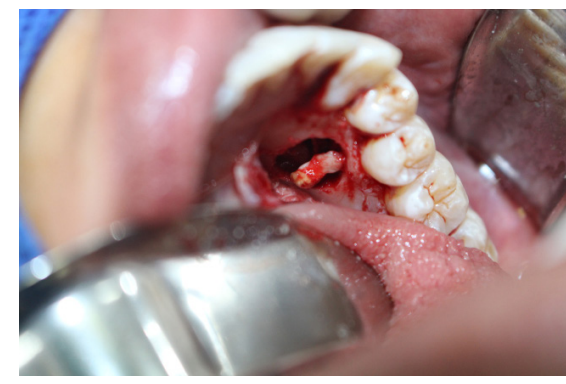

Figure 7: Osteotomy for the location of the denticles

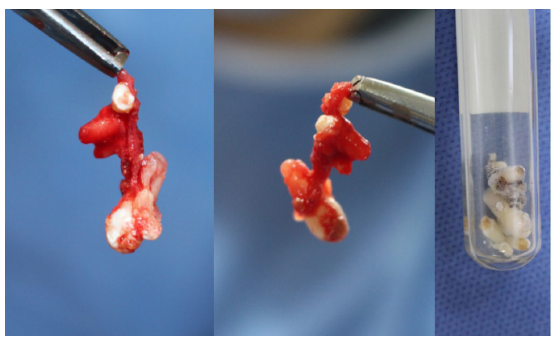

Figure 8: Enucleation of 8 denticles and their subsequent placement in a 10 -volume formalin test tube for histopathological analysis.

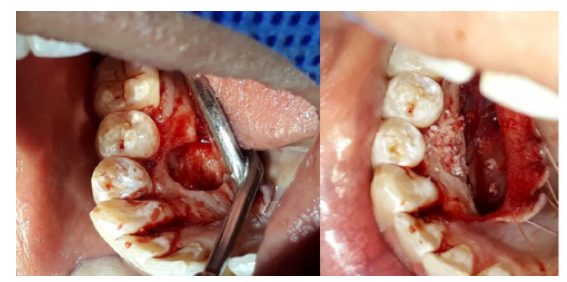

Figure 9: Cleaning of the bone cavity for subsequent placement of the bone graft previously mixed with I-PRF

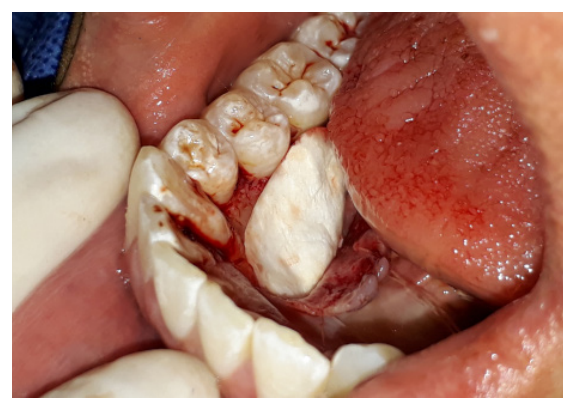

Figure 10: Resorbable collagen membrane in position. the area described above was performed (Figure 4), which reinforced the diagnosis of odontoma between the roots of 4.4 and 4.5 towards the lingual cortex; also, 6 denticles were observed, of which the most voluminous measured $10 \times 4 \mathrm{~mm}$.

With the prior consent of the patient, it was decided to perform tooth extractions of 1.8 and 3.8, together with enucleation of the lesion, followed by bone regeneration with an allograft mixed with I-PRF (injectable platelet rich fibrin) and a subsequent resorbable collagen membrane for 18 weeks.

In the intraoperative period, 4 blood tubes were obtained. By using a calibrated centrifuge for the time and revolutions per minute indicated for obtaining I-PRF, the tubes were subjected to centrifugation, extraction of I-PRF, and subsequent mixing with the allograft. (Fig. 5).

A truncal anesthesia of the posterior superior alveolar nerve, Greater Palatine, inferior alveolar, lingual and buccal nerve, using 2\% lidocaine (1:80000) was performed. Exodontia surgery was performed on teeth 1.8 and 3.8. The scalloped incision was then made without release from the mesial surface of 4.2 to the mesial surface of 4.7 (Figure 6).

The mucoperiosteal detachment was performed until insertion of the mylohyoid muscle was found. 0steotomy was performed using a carbide bur \#8 with a low-speed handpiece under the irrigation of saline solution. The bone tissue was cleared until the first denticle was found (Figure 7) and the entire lesion was completely eliminated by using elevators, hemostatic forceps and Lucas curette. Eight denticles were obtained, wrapped in fibrocollagen tissue (Figure 8) and sent for histopathological study.

The cavity was thoroughly cleaned and the bone graft combined with the I-PRF was placed in position, which was brought to the area and firmly compacted (Figure 9). In addition, a resorbable collagen membrane was placed on the graft (Figure 10 ) and the flap was repositioned by simple interrupted stitches with 5-0 polyglactin (Vicryl®). Correct hemostasis was ensured and the patient was 


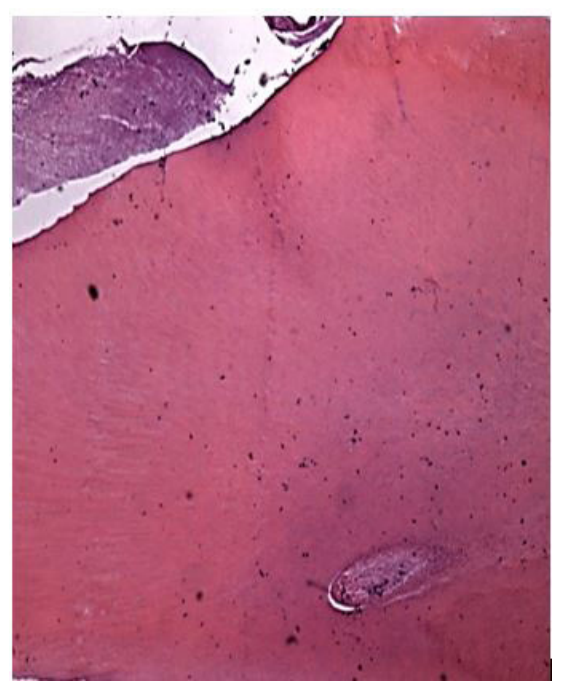

Figure 11: 40x H\&E staining, where organized dental tissue (dentin, pulp) was observed within a fibrocollagen stroma with the moderate presence of blood vessels. The definitive diagnosis was compound odontoma

medicated with Amoxicillin $+625 \mathrm{mg}$ Clavulanic Acid orally, intramuscular $75 \mathrm{mg}$ Diclofenac immediately postoperative and $600 \mathrm{mg}$ Ibuprofen orally.

The histopathological study showed organized dental tissue (enamel, dentin, pulp) within a fibrocollagen stroma with the moderate presence of blood vessels and scattered blood cells (fig.11), giving a definitive diagnosis of compound odontoma.

Postoperative controls were carried out at 24 and 72 hours, and at 8 and 15 days showing a favorable evolution and adequate healing. Radiographically, after two months, favorable bone density was observed, with no radiographic periodontal alterations of teeth 4.4 and 4.5 .

\section{DISCUSSION}

Odontomas are common tumors in the second decade of life, accounting for $75 \%$ of cases (Thistle et al., 2016). Unlike the previously cited literature, where it could be diagnosed if there was a delay in tooth eruption, this patient is in the indicated age range, but reported having had a correct chronological dental eruption.

A clear sign of the presence of an odontoma is the poor dental position and a volume increase in relation to teeth (Moya de Calderon, 2016; Palacios et al., 2016; Rojas, 2013). This agrees with this case, where the clinical examination showed a bulge in the region of the premolars, in the absence of a dental displacement.

Most of the authors state that the treatment of odontoma is surgical, with a favorable prognosis, in which an excisional biopsy can be performed (Pro Lizuain et al., 2016; Brenes-Barquero, 2014; Demicheri et al., 2015; Reddy et al., 2014; Andrade et al., 2017; Ducoing et al., 2014; Rojas, 2013), to subsequently perform the histopathological study. This case is in accordance with the literature.

The odontoma is the most common benign tumor of the oral cavity, without recurrence after a correct enucleation. In this case, surgical planning was carried out taking into account the size of the defect resulting from its enucleation, so it was decided to perform a guided bone regeneration, for a postoperative evolution and satisfactory recovery.

\section{REFERENCES}

Andrade MPA, Flores MIM, Miranda MEP, Bucheli AEI. Reabsorción apical asociada a odontoma compuesto: Reporte de caso. Odontología, 2017;18(2), 77-83.

Brenes-Barquero JL. Odontoma Compuesto: Diagnóstico Radiográfico Y Manejo Quirúrgico. Reporte De Dos Casos Clínicos. Rev Cient Odontol. 2014;9(2):39-43.

Demicheri G, Calleros S, Cacho C, Fernández A, Mazzullo A, Gehrke SA. Odontoma, revisión de la literatura a propósito de un caso clínico. Acta Odontologica. 2015;12(2):41-47

Ducoing HFJ, Olvera DJH, Reyes MJF, Aranda RS. Reporte de un caso: Hallazgo imagenologico de Odontoma Compuesto. Oral. 2014;15(47): 1093-6.

Hernández MDJD, Abreu JEA, Gómez RAM, Ramos SM, Molerio RC, Martí DMD. Odontoma compuesto. Presentación de un caso. MediCiego. 2016; 22(4):34-39.

Moya de Calderon Z. Reporte de un Caso. Diagnóstico precoz y tratamiento oportuno del odontoma compuesto de un niño Peruano de 3 años. Odontol Pediatr. 2016;14(2):155-61.

Neville B, Damm D, Allen C, Chi A. Oral and Maxillofacial Pathology. Saint Louis (M0): Elsevier, 2015.

Palacios VDE, Guzmán MB, Miranda VJE, Ramos MCA. Compound odontoma: A review of the literature and case report involving 40 denticles. Rev Asoc Dent Mex. 2016;73(4), 206-11.

Pro Lizuain C, Fungi M, Beovide V. Odontoma erupcionado: Presentación de un caso y revisión de la literatura. Odontoestomatologia. 2016;18(28):60-5.

Reddy GS, Reddy GV, Sidhartha B, Sriharsha K, Koshy J, Sultana R. Case Report Large Complex Odontoma of Mandible 
in a Young Boy: A Rare and Unusual Case Report. Case Rep Dent. 2014;2014:854986.

Rojas A. Odontología Compuesto. Rev Act Clin Med. 2013;37:1839-41.

Thistle BL, M CD, Nevarez RM, Rios BV, Nevarez RA. Descriptive aspects of odontoma: literature review. Rev Odontol Mex.
2016;20(4): 265-9.

Wright JM, Vered M. Update from the 4th Edition of the World Health Organization Classification of Head and Neck Tumours: Odontogenic and Maxillofacial Bone Tumors. Head and Neck Pathol. 2017;11(1):68-77. 